Environmental Microbiology

October 2020, Volume 22, Issue 10, Pages 4093-4095

https://doi.org/10.1111/1462-2920.15229

https://archimer.ifremer.fr/doc/00648/76040/

\title{
Vibrios - from genes to ecosystems
}

Baker-Austin Craig ${ }^{1,{ }^{*}}$, Pruzzo Carla ${ }^{2}$, Oliver James ${ }^{3}{ }^{3}$, Destourmieux-Garzon Delphine ${ }^{4}$

${ }^{1}$ Centre for Environment, Fisheries and Aquaculture (CEFAS), Weymouth, Dorset DT4 8UB, United Kingdom

2 Department of Earth, Environmental and Life Sciences (DISTAV), University of Genoa, Genoa, Italy

3 University of North Carolina, Charlotte, North Carolina, USA

4 IHPE, Université de Montpellier, CNRS, Ifremer, Université de Perpignan Via Domitia, Montpellier, France.

* Corresponding author : Craig Baker-Austin, email address : craig.baker-austin@cefas.co.uk 


\section{Editorial}

$+++++++++++++++++++++++++++++++++++++++++++++++++++++++++++++++++$

$+++++++++++++++++++$

This special edition of Environmental Microbiology "Vibrios - from Genes to Ecosystems” brings together and captures the sheer breadth of research across this fascinating genus of microorganisms, through a variety of research, opinion and review articles. Perhaps one of the most captivating aspects related to vibrios is the duality that they represent in microbiology. On one hand they are important autochthonous constituents of marine and coastal waters throughout the world, playing critical ecological roles through a myriad of phylogenetically and functionally diverse representatives; on the other, unfortunately, vibrios constitute a uniquely potent group of human and animal pathogens capable of causing an array of diseases such as gastroenteritis, sepsis, and wound infections. Through the most famous (or infamous) member of this genus - V. cholerae, cholera has remained a global scourge, and alongside the seafood pathogen $V$. parahaemolyticus, the only pathogens that have emerged from aquatic and marine sources to cause bona fide global pandemics. Certainly, the research studies presented in this special edition draw on many aspects of this duality and provide critical insights regarding the growing importance of these bacteria, perhaps in the process highlighting that vibrios constitute a separate field of study in and of itself.

Several review articles in this special edition highlight why these bacteria are a critically important topic to study in microbiology. Vezzulli et al. (2020) outlines the growing 


\section{Editorial}

importance of non-cholera V. cholerae (non O1/O139) using a variety of epidemiological, microbiological and public health data, and asks the pertinent question if this member of the Vibrionaceae family deserves greater research emphasis, particularly given the impact of climate change on this important human pathogen. Similarly, Daines and Froelich (2020) outline how and why a warming climatic system may place humans in greater contact - and consequently risk, of pathogenic vibrios. The risk of contamination is also explored by Canty et al. (2020) who study the effect of farming practices on the prevalence of the human pathogen $V$. vulnificus in oysters. Moreover, the review article by Hernández-Cabanyero and Amaro (2020) provides insights into the evolution and life strategies of this pathogen in response to selected environmental parameters. This article also contributes to fill an important gap in the existing knowledge on the biology of $V$. vulnificus and stresses its importance as a zoonotic pathogen linked to fish farms and climate change.

Several aspects related to the fascinating biology and genomics of vibrios are described here too. Sozhamman and Waldminghaus (2020) outline the importance of dual chromosomes in vibrios - focussing on the pathogen $V$. cholerae. Single-chromosome $V$. cholerae have been previously identified, and these particular strains are essential to study fundamental questions on chromosome biology in general, and DNA replication in particular. Furthering our understanding of the biology of these organisms, Crisan and Hammer (2020) provide a cogent overview of the importance of the type 6 secretions system (T6SS) as proteinaceous weapons used by a variety of Gram-negative bacteria. Utilizing $V$. cholerae as an exemplar model organism for T6SS they reveal the broad yet growing importance of these secretion 


\section{Editorial}

systems as intrinsic mechanisms to evade phagocytic eukaryotes, cause intestinal inflammation, and compete against other bacteria with toxins that disrupt lipid membranes, cell walls and actin cytoskeletons.

Recently, Vibrio bacteria have also garnered attention as host for molecular biology and biotechnology applications. As outlined in the review article by Hoff et al. (2020), this is the case with $V$. natriegens, the fastest-growing non-pathogenic bacterium known to date. Spanning all the way from its initial isolation to its latest applications, the article focuses on $V$. natriegens successful applications in synthetic biology and biotechnology in recent years, and examines missing methods and tools which need to be established in order to exploit the full potential of this fascinating bacterium.

Identifying the mechanisms underpinning virulence traits and understanding the drivers of their selection is key for the prevention of human and animal diseases caused by vibrios. Here are a series of articles that study virulence determinants in environmental and clinical strains. The article by Piel et al. (2019) reveals the importance of a plasmid highly prevalent in oyster farms, which encodes a T6SS involved in virulence in the oyster pathogen, $V$. crassostreae. While the T6SS contributes to the dampen the immune defenses of the oyster, Drebes Dörr and Blokesch (2020) show that T6SSs in environmental isolates of $V$. cholerae are major players of interbacterial competition while others, which harbor an actin cross-linking domain on the VgrG protein, and confer resistance to amoeba grazing. Seibt et al. (2020) explore the role of the 654 -dependent transcriptional regulator VasH in the expression of such secretion 


\section{Editorial}

system in pandemic cholera causing El Tor strain A1552, which requires specialized growth signals of low temperature and high osmolarity for T6SS expression. A study presented here by Stutzmann et al. (2020) compared the chitin-induced competence and transformation behavior in a variety of pandemic O1 El Tor V. cholerae isolates as well as an $\mathrm{O} 139$ isolate. Using a variety of methodologies, including molecular microbiological approaches, sequencing and comparative bioinformatics they demonstrate both the integral role of the competence regulon as well as conserved nature of this system in pathogenic $V$. cholerae, further outlining the role of chitin and HGT in this context.

Two articles show that the multiple host and environmental stressors acting on the selection of Vibrio virulence factors may lead to cases of conflicting or coincidental selection. In $V$. splendidus, Oyanedel et al. (2020) show that the structure of the O-antigen is detrimental for virulence but essential for resistance to amoeba grazing, which likely explains it hypervariability in environmental isolates. In contrast, the article by Robino et al. (2019) shows that resistance to copper and toxic factors (metalloproteases) in $V$. tasmaniensis are beneficial in the interaction with both oysters and marine amoeba. How the interactions of vibrios with environmental predators and metazoan hosts may be beneficial or costly for Vibrio virulence is discussed in Destoumieux-Garzón et al. (2020), who review the current knowledge on Vibrio-bivalve interactions. Critically important to understand how and why these bacteria can cause infections in humans and animals is the need for reliable, tractable and repeatable model systems, and especially those that constitute and reflect real exposure hazards. Hubert and Michell (2020) outline an approach to generate 'artificial' marine snow, 


\section{Editorial}

into which bacteria such as $V$. vulnificus can be incorporated to facilitate extensive uptake by oysters. This represents a highly promising and adaptable model for bacterial study within filter-feeding shellfish - the major route through which many of these species cause disease in humans. This study also outlines the growing importance of the T6SS in terms of potentially modulating numbers of these pathogens in the environment, critically important information to more fully understand the ecology of $V$. vulnificus.

Other articles of this special edition tackle the vast complexity of Vibrio biology and ecology highlighting how science is moving on fast in this fascinating research field. The opinion paper by Baker-Austin et al. (2020) outlines some of the most exciting, innovative and translational scientific advances that are currently being applied to these ecologically, environmentally and clinically important bacteria. From this paper, as well as from the other contributions of the special edition, it is evident that the coordinated use of different experimental approaches across various disciplines, not just microbiological, is the most fruitful strategy to decipher structures and mechanisms underlying the duality of life style of Vibrio bacteria, and to clarify the still obscure aspects of their interactions with both the environment and their host. We feel that the enormous breadth of research captured in this special edition - encompassing microbiology, molecular biology, genetics, and bioinformatics, among others - outline the true interdisciplinary nature of vibrios, and underline why they continue to represent such a fascinating and rewarding group of microorganisms to study. 


\section{Editorial}

\section{References}

Baker-Austin, C., Trinanes, J., and Martinez-Urtaza, J. (2020) The new tools revolutionizing Vibrio science. Environ Microbiol doi: 10.1111/1462-2920.15083.

Canty, R., Blackwood, D., Noble, R., and Froelich, B. (2020) A comparison between farmed oysters using floating cages and oysters grown on-bottom reveals more potentially human 


\section{Editorial}

pathogenic Vibrio in the on-bottom oysters. Environ Microbiol doi: 10.1111/14622920.14948.

Crisan, C.V., and Hammer, B.K. (2020) The Vibrio cholerae type VI secretion system: toxins, regulators and consequences. Environ Microbiol doi: 10.1111/1462-2920.14976.

Destoumieux-Garzón, D., Canesi, L., Oyanedel, D., Travers, M.A., Charrière, G.M., Pruzzo, C., and Vezzulli, L. (2020) Vibrio-bivalve interactions in health and disease. Environ Microbiol doi: 10.1111/1462-2920.15055.

Drebes Dörr, N.C., and Blokesch, M. (2020). Interbacterial competition and anti-predatory behavior of environmental Vibrio cholerae strains. Environ Microbiol https://doi.org/10.1111/1462-2920.15224

Froelich, B.A., and Daines, D.A. (2020) In hot water: effects of climate change on Vibriohuman interactions. Environ Microbiol doi: 10.1111/1462-2920.14967

Hernández-Cabanyero, C., and Amaro, C. (2020) Phylogeny and life cycle of the zoonotic pathogen Vibrio vulnificus. Environ Microbiol doi: 10.1111/1462-2920.15137.

Hoff, J., Daniel, B., Stukenberg, D., Thuronyi, B.W., Waldminghaus, T., and Fritz, G. (2020) Vibrio natriegens: an ultrafast-growing marine bacterium as emerging synthetic biology chassis. Environ Microbiol doi: 10.1111/1462-2920.15128.

Hubert, C.L., and Michell, S.L. (2020) A universal oyster infection model demonstrates that Vibrio vulnificus Type 6 secretion systems have antibacterial activity in vivo. Environ Microbiol doi: 10.1111/1462-2920.15123.

Oyanedel, D., Labreuche, Y., Bruto, M., Amraoui, H., R,obino, E., Haffner, P., Rubio, T., Charrière, G.M., Le Roux, F., and Destoumieux-Garzón, D. (2020) Vibrio splendidus Oantigen structure: a trade-off between virulence to oysters and resistance to grazers. Environ Microbiol doi: 10.1111/1462-2920.14996.

Piel, D., Bruto, M., James, A., Labreuche, Y., Lambert, C,, Janicot, A., Chenivesse, S., Petton, B., Wegner, K.M., Stoudmann, C., Blokesch, M., and Le Roux, F. (2019) Selection of Vibrio crassostreae relies on a plasmid expressing a type 6 secretion system cytotoxic for host immune cells. Environ Microbiol doi: 10.1111/1462-2920.14776.

Robino, E., Poirier, A.C., Amraoui, H., Le Bissonnais, S., Perret, A., Lopez-Joven, C., Auguet, J.C., Rubio, T.P., Cazevieille, C., Rolland, J.L., Héchard, Y., Destoumieux-Garzón, D., and Charrière, G.M. (2019) Resistance of the oyster pathogen Vibrio tasmaniensis LGP32 against grazing by Vannella sp. marine amoeba involves Vsm and CopA virulence factors. Environ Microbiol doi: 10.1111/1462-2920.14770. 


\section{Editorial}

Seibt, H,. Aung, K.M., Ishikawa, T., Sjöström, A., Gullberg, M., Atkinson, G.C., Wai, S.N., and Shingler, V. (2020) Elevated levels of VCA0117 (VasH) in response to external signals activate the type VI secretion system of Vibrio cholerae O1 El Tor A1552. Environ Microbiol doi: 10.1111/1462-2920.15141.

Sozhamannan, S., and Waldminghaus, T. (2020) Exception to the exception rule: synthetic and naturally occurring single chromosome Vibrio cholerae. Environ Microbiol doi: 10.1111/1462-2920.15002.

Stutzmann, S., and Blokesch, M. (2020) Comparison of chitin-induced natural transformation in pandemic Vibrio cholerae O1 El Tor strains. Environ Microbiol https://doi.org/10.1111/1462-2920.15214

Vezzulli, L., Baker-Austin, C., Kirschner, A., Pruzzo, C., and Martinez-Urtaza, J. (2020) Global emergence of environmental non-O1/O139 Vibrio cholerae infections linked with climate change: a neglected research field? Environ Microbiol doi: 10.1111/14622920.15040 . 